\title{
Appendiceal endometriosis: a greater mimicker of appendicitis
}

\author{
David H Jeong *, MD, Hyojin Jeon, MD, MPH, Karen Adkins, MD \\ Department of Surgery, Trinity School of Medicine, Ribishi, Saint Vincent \\ *Corresponding author: kb_1991@hotmail.com
}

Hong Kong Med J 2019;25:492-3

https://doi.org/10.12809/hkmj187654

Endometriosis is defined as the presence of functioning endometrial glandular cells outside the uterine cavity. It is a common cause of pelvic pain that is worse during the menstruation cycle. Endometriosis is extremely difficult to diagnose based on clinical features, because the location of the ectopic endometrial cells could lead to diagnosis of other common pathological causes of abdominal pain specific to that area. Although some female patients remain asymptomatic, endometriosis is usually associated with dysmenorrhea, chronic pelvic pain, and infertility and can lead to bowel obstruction or abdominal mass. ${ }^{1}$

Although endometriosis within the uterine muscle wall is commonly seen in about $10 \%$ of women of menstrual age, ectopy of endometrial cells into the appendix is rare. The true prevalence of extragenital endometriosis is unclear, owing to the lack of studies or cases; the incidence of appendiceal endometriosis has been reported to be as low as $0.054 \%$ and as high as $0.8 \% .^{2}$ Although endometriosis has been reported in almost any part of the human body, to the best of our knowledge no cases have yet reported endometriosis in the spleen. ${ }^{3}$

Appendiceal endometriosis is diagnosed pathologically. The presence of glandular tissue, endometrial stroma, and haemorrhage are typical findings in patients with endometriosis regardless of the location. ${ }^{4}$ There is no correlation between the location of the endometriotic foci and clinical symptoms $^{5}$ and endometriosis is much likely to mimic primary inflammatory diseases. In the patient described in this case report, ectopic endometrial cells mimicked inflammation inside the appendiceal cavity and the patient presented with clinical symptoms that were consistent with acute appendicitis.

In 2018, we experienced a 34-year-old woman who presented with right lower quadrant abdominal pain for 1 day. The patient described the localised pain as crampy and rated the pain severity as " 10 " on a scale of 1 to 10 . The patient presented with nausea, vomiting, headache, constipation, menorrhagia, and dizziness. She also reported that she was actively menstruating and that these symptoms typically occurred monthly with menstruation, but had been particularly severe in that month. The patient has two children and reported not using any form of contraception. The patient's medical history included inflammatory bowel disease, migraine, chronic lower back pain, and asthma. The patient had a Caesarean section delivery in 2000 and left fallopian tube/ovary removal secondary to ruptured ectopic pregnancy in 2008. The patient denied use of alcohol, tobacco, or illicit drugs. There was no relevant family history of gastrointestinal diseases or malignancies and the patient was allergic to morphine and azithromycin.

Physical examination showed mild distension of the abdomen and tenderness to palpation on both right and left lower quadrants. No rebounding tenderness or guarding was noted. Vital signs and laboratory test results were all within normal limits with the exception of slightly elevated white blood cell at $11.69 \mathrm{~K} / \mathrm{mm}^{3}$. Although $5.32 \mathrm{M} / \mathrm{mm}^{3}$ of red blood cell was observed in the urinalysis, the results were considered within normal limits since the patient was actively menstruating at the time of the test. Computed tomography scan of the patient's abdomen and pelvis (Fig 1) showed thickwalled appendix $(>7 \mathrm{~mm})$ consistent with acute appendicitis.

The patient was taken to the operation room for laparoscopic appendectomy. When the laparoscope was inserted inside the patient's abdominal cavity, significant adhesion of the entire abdominal wall was noted. The appendix was unable to be visualised even after lysis of adhesion was attempted using electrocautery. A decision was made to proceed to open appendectomy. Further lysis of adhesions had to be done to visualise the appendix, which did not show any gross inflammation. The appendix was excised and sent to pathology lab for further investigation.

The appendix did not show any signs of inflammation, and the preoperative diagnosis of acute appendicitis was changed to possible endometriosis or ruptured cyst. However, 3 days after the appendectomy, pathology results showed infiltration of endometrial glands, endometrial stroma, and blood into the appendix (Fig 2). On the basis of these findings, appendiceal endometriosis was finally diagnosed. After the appendectomy, the 
patient reported substantial improvement of the right lower quadrant pain.

Appendiceal endometriosis is rare and its preoperative diagnosis based on clinical features and/or imaging is extremely difficult. Differential diagnosis in female patients who present with acute pain in the right lower quadrant, especially those who are of menstruating age, should include appendiceal endometriosis. Laparoscopy is useful for the diagnosis since no gross inflammation is observed in the appendix itself and appendectomy relieves the acute symptoms.

\section{Author contributions}

All authors contributed to the concept of study, acquisition and analysis of data, drafting of the article, and critical revision for important intellectual content. All authors had full access to the data, equally contributed to the study, approved the final version for publication and take responsibility for the accuracy and integrity of the content.

\section{Conflicts of interest}

The authors have no conflicts of interest to disclose.

\section{Funding/support}

This research has received no specific grant from any funding agency in the public, commercial, or not-for-profit sectors.

\section{Ethics approval}

The patient was treated in accordance with the Declaration of Helsinki. The patient provided informed consent for all procedures.

\section{References}

1. Yantiss RK, Clement PB, Young RH. Endometriosis of the intestinal tract: a study of 44 cases of a disease that may cause diverse challenges in clinical and pathological evaluation. Am J Surg Pathol 2001;25:445-54.

2. Collins DC. A study of 50,000 specimens of the human vermiform appendix. Surg Gynecol Obstet 1955;101:43745 .

3. Berker B, Lashay N, Davarpanah R, Marziali M, Nezhat CH, Nezhat C. Laparoscopic appendectomy in patients with endometriosis. J Minim Invasive Gynecol 2005;12:206-9.

4. Apostolidis S, Michalopoulos A, Papavramidis TS, Papadopoulos VN, Paramythiotis D, Harlaftis N. Inguinal

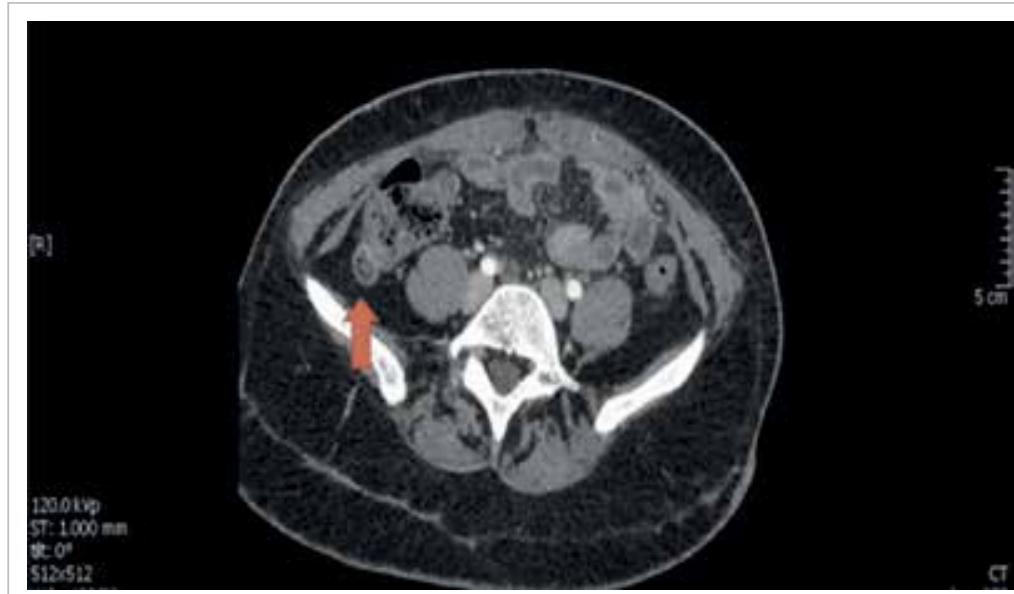

FIG I. Thickening of the walls of the appendix (orange arrow)

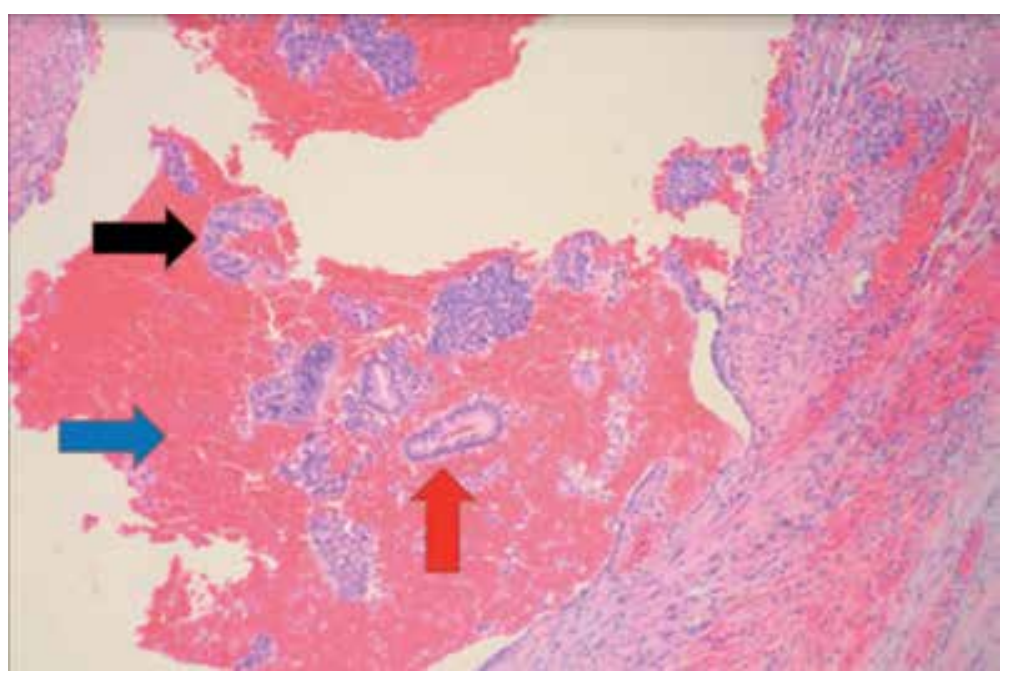

FIG 2. Endometrial stroma (black arrow), endometrial gland (red arrow), and blood (blue arrow)

endometriosis: three cases and literature review. South Med J 2009;102:206-7.

5. Uncu H, Taner D. Appendiceal endometriosis: two case reports. Arch Gynecol Obstet 2008;278:273-5. 